\title{
Development of Integrated Framework for the High Temperature Furnace Design
}

\author{
Yu Xuan Jin ${ }^{1}$, Jae-Woo Lee ${ }^{2, *}$ Karp Joo Jeong ${ }^{3}$, Jong Hwa Kim ${ }^{4}$, \\ and Ho-Yon Hwang 5 \\ ${ }^{1}$ Graduate Research Assistant, Department of Advanced Technology Fusion \\ ${ }^{2}$ Professor, Department of Aerospace Engineering \\ jwlee@konkuk.ac.kr \\ ${ }^{3}$ Professor, Department of Internet \& Multimedia Engineering \\ ${ }^{4}$ Professor, Department of Industrial Engineering \\ ${ }^{5}$ Professor, Department of Aerospace Engineering, Sejong University, \\ Center for Advanced e-System Integration Technology (CAESIT) \\ Konkuk University, Seoul 143-701, Republic of Korea
}

\begin{abstract}
In this research the high temperature vacuum furnace design framework with centralized database system, distributed middleware and integrated graphic user interface(GUI), are developed with configuration module(CAD), thermal analysis module, optimization modules, and database management system. By considering the operation under the distributed computing environment, the method of managing the analysis modules and the optimization process is proposed and the integration method of database system and CAD system under local PC environment is demonstrated by carrying out example design problem.
\end{abstract}

\section{Introduction}

For the product design and development of the most small and medium enterprises, the design, analysis and the production department are geographically and business wise separated. Various software programs are scattered and need integration for the efficient design and development. Therefore, dissimilar and distributed computational environment and resources must be integrated seamlessly. Because the high temperature vacuum (gas) furnace requires elaborate technology, low production cost, and fast design cycle, an integrated design framework must be developed which can integrate the database operation, the design automation, and the integrated analysis.

General process of furnace design may start with user's requirements, such as target temperature, ambient gas and uniform temperature zone size. Materials and sizes of heater and insulator are decided based on the user's requirements. Then, the chamber size and type are determined. Thereafter, the vacuum system, the temperature control system and the cooling system are consequently determined. Finally, a sample-loading type is decided. Figure 1 shows the design process of a vacuum furnace.[1]

\footnotetext{
* Corresponding author.
} 


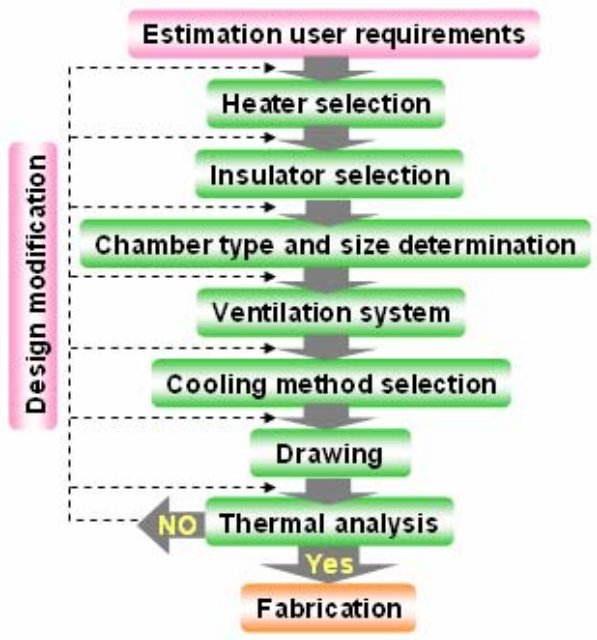

Fig. 1. Design Process

Since the design process is empirical and executed one after another, design issues that occur in the middle of the design or manufacturing can not be properly accounted. If the problems are not resolved within the design stage, whole design process must be repeated again. When the problems occur at the production stage, the development time and cost may be increased.

Existing method of design vacuum furnace is a trial and error method, which before producing vacuum furnace it is impossible to make an estimation performance. And it depends on experience. So when we produce vacuum furnace expense too much cost and it is not efficient. Consequently development of the efficient vacuum design technique with the optimization is necessary and the design framework which provides development environment is necessary. Utilizing design function user can judge the result with support data from GUI interface in the related design at the same time[2].

In this research the high temperature vacuum furnace design framework with centralized database system, distributed middleware and integrated graphic user interface(GUI), shall be developed with configuration module(CAD), thermal analysis module, optimization modules, and database management system.

\section{Integration Architecture of High Temperature Vacuum Furnace Design Framework}

To obtain the framework architecture under the distributed computing environment, framework's requirements and commercial framework software's architecture are to be analyzed. Table 1 shows framework requirements[3].

The framework development is summarized with two objectives. First, it is a design function which can efficiently accomplish MDO (Multidisciplinary Design 
Optimization) at time and cost. Second, it is an integration design environment that the field experts can participate in design at the same time. The framework meaning of expandability is a possibility of trying to observe from two viewpoints. First, it is the expandability that framework can apply design subject which is possible. Second, it is the expandability that framework can add like analysis optimization codes which is possible. Because framework aims an independent design environment for design subject, link method and data flow of code will not be able to apply with fixed pattern.

Table 1. Framework Requirements

\begin{tabular}{|c|c|}
\hline $\begin{array}{c}\text { Basic } \\
\text { Requirements }\end{array}$ & $\begin{array}{l}\text { CAD/CAE tools integration method } \\
\text { Management of analysis code data flow } \\
\text { Management of analysis and optimization process } \\
\text { Grasp the function in GUI }\end{array}$ \\
\hline $\begin{array}{c}\text { Essential } \\
\text { Requirement }\end{array}$ & $\begin{array}{l}\text { - Execute under the heterogeneous distributed environment } \\
\text { - Centralized DBMS (Database Management System) } \\
\text { - Metecting wrong data flow and process } \\
\text { - Delete, add, modify the design process } \\
\text { - Create design variables for the complex design problem } \\
\text { - Restart at wrong design point } \\
\text { The object oriented design for an expandability and standard } \\
\text { development tool and implementation }\end{array}$ \\
\hline $\begin{array}{c}\text { Additional } \\
\text { Requirements }\end{array}$ & $\begin{array}{l}\text { Manage execution with scheduler } \\
\text { Parallel computing }\end{array}$ \\
\hline
\end{tabular}

The framework which considers expandability in distributed computing environment, the design plan is materializing design, analysis resources as 'component', and locating above the distributed environment system Layer which takes charge of a distributed environment. Considering this requirement, the high temperature vacuum furnace design framework centralizes distributed system(PLinda), integrated graphic user interface, configuration, database, analysis, and optimization modules. The figure 2 shows the development concept in high temperature furnace design framework.

\subsection{High Temperature Vacuum Furnace Design Framework System Architecture}

The high temperature vacuum furnace design framework was constructed of 6 modules. Using distributed middleware for integrating distributed resource, select Microsoft Access for managing data, and selecting Visual Basic language are easy to 


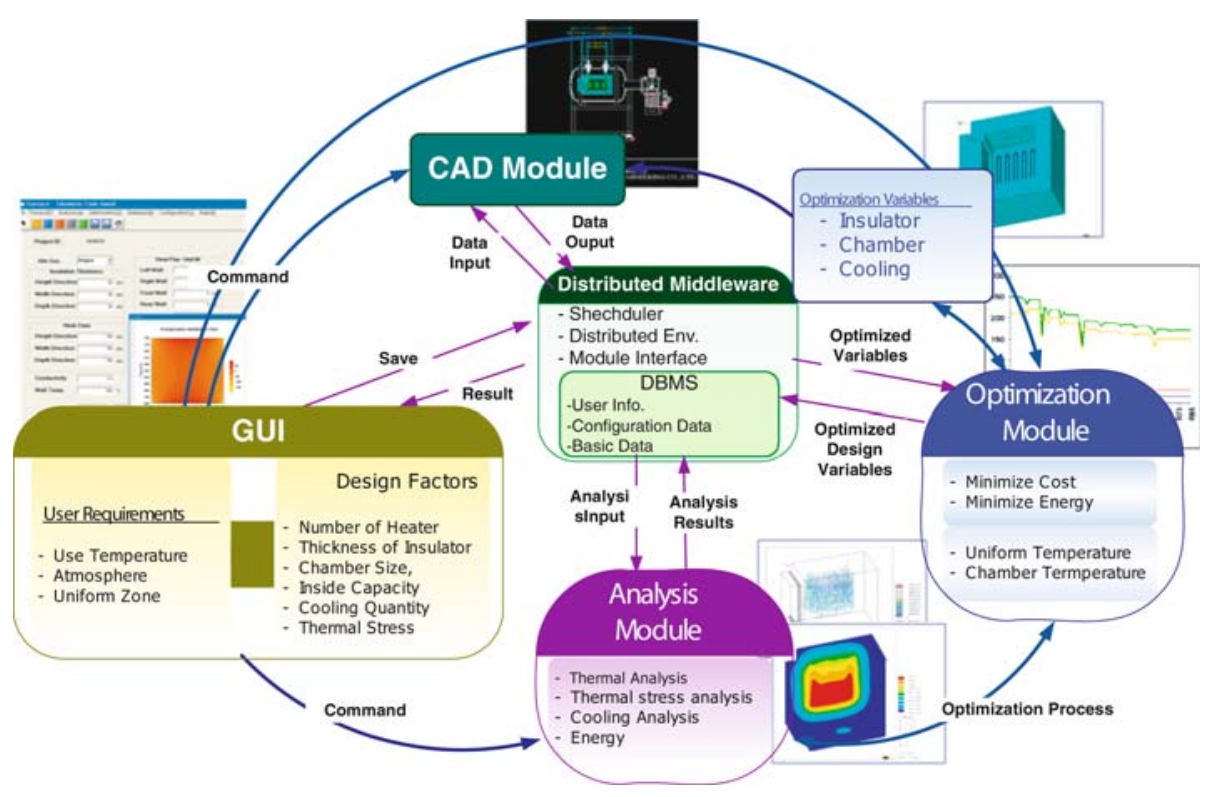

Fig. 2. Framework Development Concept[4]

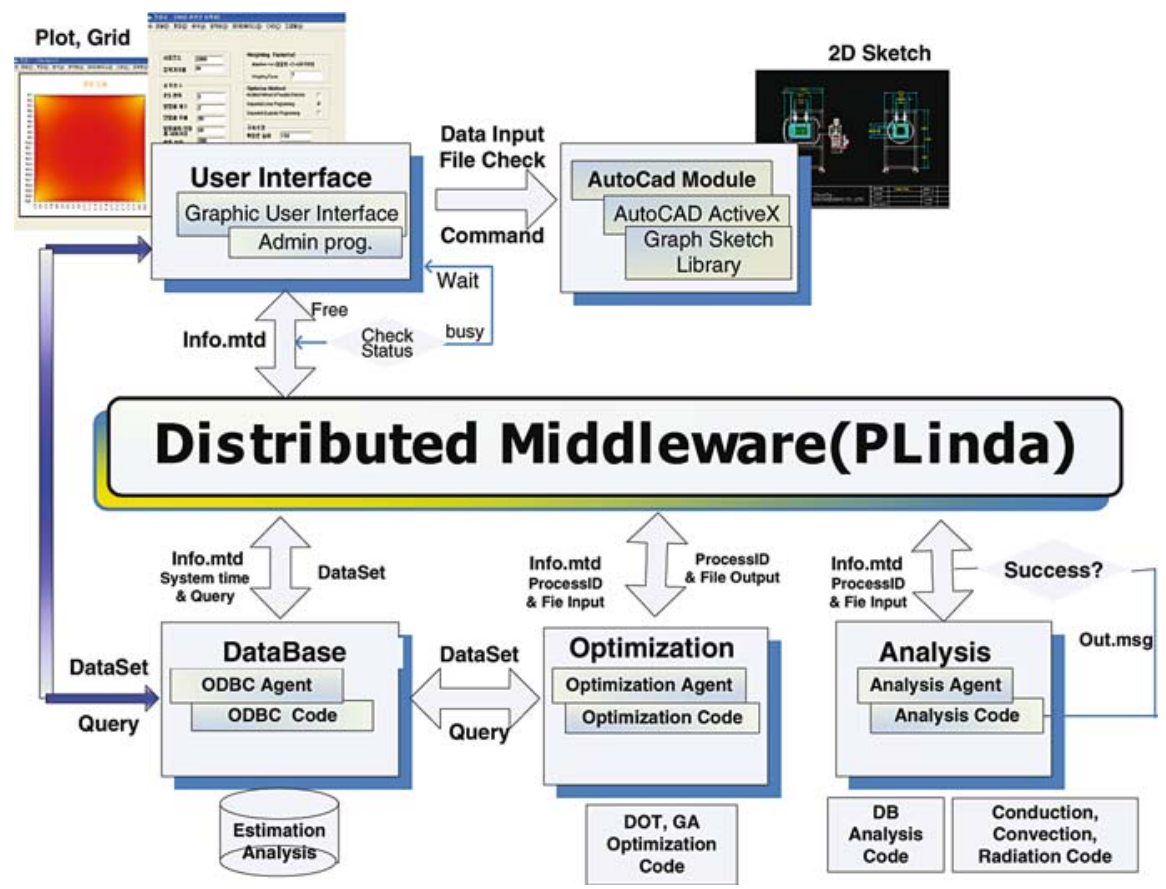

Fig. 3. System Integration Architecture 
develop GUI. Selecting the AutoCAD for drawing vacuum furnace configuration, and using DOT optimization Code(Gradient Method), thermal analysis code including conduction, convection, radiation. The accuracy of analysis code can be confirmed in final report of 'Development of High Temperature Vacuum Furnace Through Integrated Design and Optimization Technology'. The figure 3 shows system integration architecture [5].

\subsection{High Temperature Vacuum Furnace System Design Framework}

\section{Integration Plan of Analysis Code}

So far most integration method of analysis code is developed using I/O file. Analysis codes which only offers executable program in ultra high temperature vacuum furnace system are integrated using the in/output files and mixed language is employed when the source file is available. For the integration using analysis code's I/O, standard I/O metadata file and analysis code input file based on the parameter defined by the user are created. I/O file is displayed on GUI and is saved according to the order of user's selection and data type. Figure 4 is the structure of code integration using file $\mathrm{I} / \mathrm{O}$.

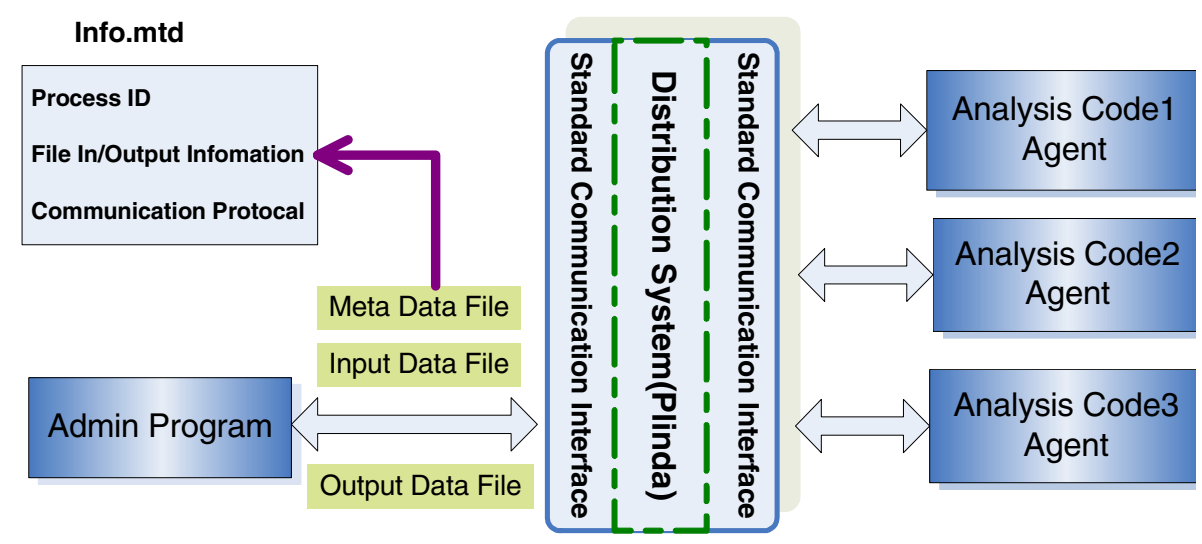

Fig. 4. Analysis Code Integration Architecture

Because the integration of analysis code is realized through file's I/O in ultra high temperature vacuum furnace system program, if analysis code's I/O is not changed, it required that a new version of analysis code is modified even if it is changed.

\section{Integration of Optimization Code}

When formulating the vacuum furnace system optimization problem, we read existing database are drawn and carried out for the integration. In many cases the optimization codes are written by Fortran[6]. As the Fortran language can not directly access the database, C++ and Fortran are used together. Figure 5 shows the integration concept of the optimization code and the analysis modules. 


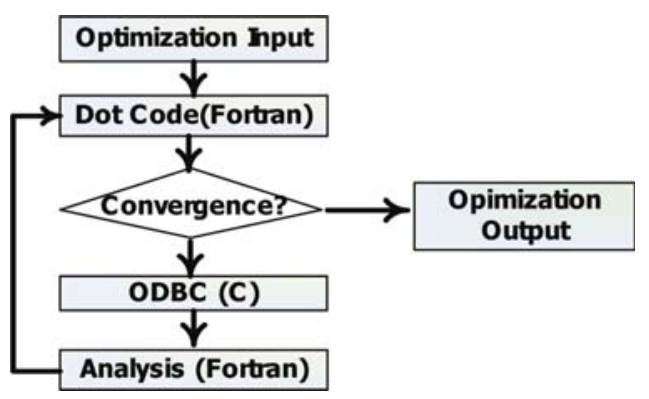

Fig. 5. Optimal Code Integration Architecture

\section{Integration of Database}

There were two kinds of methods in this system for integrating database system. First method uses file's I/O. Though analysis code is same with the integration method, input file is realized in query and data values. When user needs data for executing analysis code or optimal data, after create query and connect database, user can draw out any data which user wants. Second method doesn't directly pass middleware from user interface GUI and input data or read data about vacuum furnace system products through connecting database. Figure 3 shows the integration structure of database.

\section{Integration of CAD}

AutoCAD[7] cannot go through the distributed middleware for designing system image, but realized GUI and integration directly. Figure 6 is the integration structure of CAD and GUI. Standard vacuum furnace system design map was used and image data was drawn out in AutoCAD. When vacuum furnace design using database reaches optimal design, the fixed map make full use of AutoCAD Block function to express detailed CAD image completely using seven design parameters.

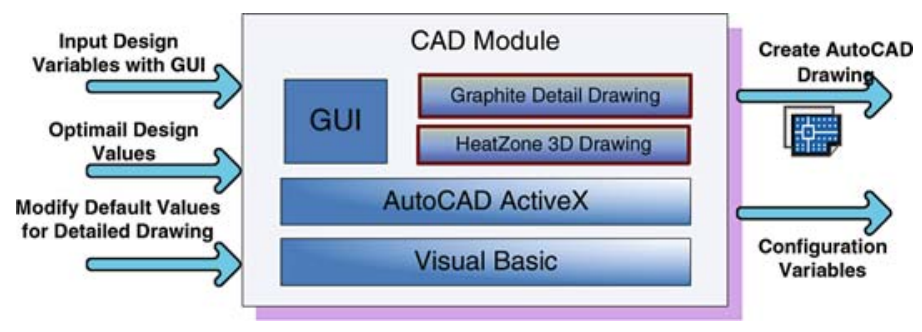

Fig. 6. AutoCAD Integration Architecture

\section{Experiments}

High temperature vacuum furnace design framework was developed on November 2004, which is now being used for actually furnace design and manufacturing. The following figures are the optimized result extracted by the $\mathrm{iFuD}$ (intelligent Furnace 
Designer) system[4]. The first figure is the data representation by visualization toolFormula One. The data is represented by the form of graph, contour and data grid. The fourth figure shows the final configuration of furnace represented by AutoCAD program.

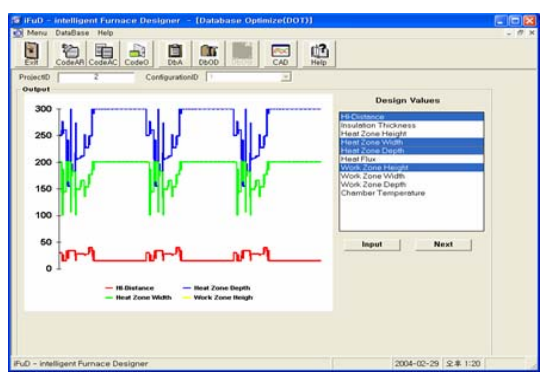

Fig. 7. Plot and Display -Graph

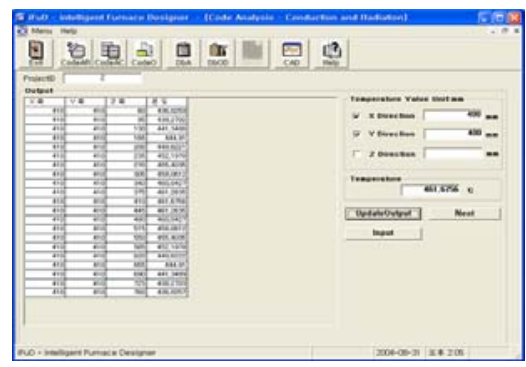

Fig. 9. Plot and Display -Data Grid

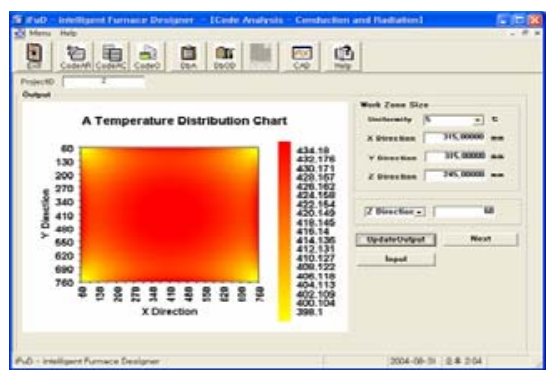

Fig. 8. Plot and Display -Contour

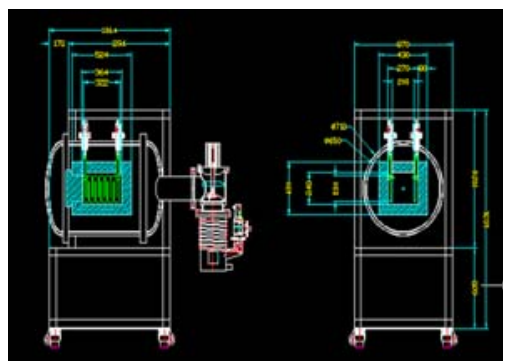

Fig. 10. Configuration for Manufacturing

\section{Conclusions}

From the research of high temperature design framework development, following conclusions are made.

First, by utilizing the centralized database management system to manage and integrate design data, and by using the distributive middle ware, each design analysis module is integrated through the object oriented concept. Then, high temperature design framework is developed by considering the expandability and easy modification

Second, the analysis codes are integrated using the in/output files and mixed language is employed when the source file is available.

Third, by considering the operation under the distributed computing environment, the method of managing the analysis modules and the optimization process is proposed and the integration method of database system and CAD system under local PC environment is demonstrated by carrying out example design problem. 


\section{References}

1. Min-Ji Kim, Ho-Girl Jung, Jae-Woo Lee, Changjin Lee and Yung-Hwan Byun, "Optimal Design of an Energy Efficient Vacuum Furnace Using Thermal Analysis Database," Key Engineering Materials, Vols 277-279, pp 732-740, Jan. 2005.

2. Sang-Oh Cho, Jae-Woo Lee, and Yung-Hwan Byun, "A Study on the Integration of Analysis Modules and Optimization Process in the MDO Framework," KSAS Journal, Vol. 30, No. 7, Oct. 2002.

3. Salas, J. C. Townsend, "Framework Requirements for MDO Application Development, AIAA-98-4740. 1998.

4. Jae-Woo Lee, "Development of High Temperature Vacuum Furnace Through Integrated Design and Optimization Technology," Final Report, Jun. 2004.

5. Shenyi Jin, Kwangsik Kim, Karp-Joo Jeong, Jae-Woo Lee, Jong-Hwa Kim, Hoyon Hwang, Hae-Gok Suh, "MEDIC: A MDO-Enabling Distributed Computing Framework", Lecture Notes in Artificial Intelligence, Vol. 3613 part 1, Springer-Verlag, pp. 1092-1101, Aug. 2005.

6. DOT Users Manual, Vanderplaats Researtch \& Development Inc., 1995.

7. AutoCAD User Manual(ActiveX and VBA Developer's Guide), Autodesk Inc., 2002. 